\title{
Three-Dimensional Ordered Porous Carbon for Energy Conversion and Storage Applications
}

\author{
Jinxiu Feng ${ }^{1}$, Dong Zheng ${ }^{1}$, Xinlong Gao ${ }^{1}$, Wenbin Que ${ }^{1}$, Wenhui Shi ${ }^{2 *}$, Wenxian Liu', \\ Fangfang $W^{1}$ and Xiehong $\mathrm{Cao}^{1 *}$ \\ ${ }^{1}$ College of Materials Science and Engineering, Zhejiang University of Technology, Hangzhou, China, ${ }^{2}$ Center for Membrane \\ Separation and Water Science \& Technology, Zhejiang University of Technology, Hangzhou, China
}

OPEN ACCESS

Edited by:

Teng Zhai,

Nanjing University of Science and Technology, China

Reviewed by:

Xihong Lu,

Sun Yat-sen University, China

Xinhui Xia,

Zhejiang University, China

*Correspondence:

Wenhui Shi

shiwh@zjut.edu.cn

Xiehong Cao

gcscaoxh@zjut.edu.cn;

caoxiehong@gmail.com

Specialty section:

This article was submitted to Electrochemical Energy Conversion and Storage,

a section of the journa

Frontiers in Energy Research

Received: 29 June 2020 Accepted: 05 August 2020

Published: 27 August 2020

Citation:

Feng J, Zheng D, Gao X, Que W Shi W, Liu W, Wu F and Cao X (2020) Three-Dimensional Ordered Porous

Carbon for Energy Conversion

and Storage Applications.

Front. Energy Res. 8:210.

doi: 10.3389/fenrg.2020.00210
The performance of energy storage devices is highly related to the properties of electrode materials, such as components, morphology, configurations and so on. As a typical hierarchical carbon material, three-dimensional ordered porous carbon (3DOPC) has unique characteristics of low cost, large specific surface area, highly ordered channels, and high electronic and ionic conductivity, which shows great potential in energy storage and conversion applications. In this mini review, we summarize various template-assisted preparation methods for 3D-OPC, including hard-, ice- and self-templated approaches, and their applications in electrocatalysis, batteries and supercapacitors. Additionally, the critical roles of vertical channels in 3D-OPC when used as electrodes are also discussed. Finally, the current challenges and future research outlook of 3D-OPC are proposed.

Keywords: 3D ordered porous carbon, energy storage and conversion, vertical channels, template-assisted methods, low tortuosity

\section{INTRODUCTION}

Developing safe, low-cost and efficient energy storage and conversion devices has become an urgent need, owing to the growing demands of sustainable resources, such as solar and wind power (Cao et al., 2017; Li et al., 2020; Wu M. et al., 2020). Carbon materials exhibit numerous advantages, such as high electrical conductivity, low cost and high specific surface area (Cao et al., 2014; Li et al., 2019; Wu Q. et al., 2020). Carbon materials can be categorized into four types, i.e., zerodimensional (0D, e.g., $\left.\mathrm{C}_{60}\right)$, one-dimensional (1D, e.g., carbon nanofibers and carbon nanotubes), two-dimensional (2D, e.g., graphene and carbon sheets), and three-dimensional structures (3D, e.g., 3D porous carbon and graphene foam) (Zhang F. et al., 2017). In recent years, 3D carbon materials have demonstrated excellent electrochemical performance in a wide range of applications including energy storage and conversion (Ullah et al., 2019; Ni and Li, 2020).

In a conventional route for preparation of a certain electrode, a slurry composed of active material, conductive agent and binder is normally casted onto a current collector. With increase of the loading mass of active material and the thickness of electrode, its electrical conductivity decreases. Besides, thick electrode leads to high tortuosity, which hinders the penetration of electrolyte and ion transport and results in limited utilization of active materials (Wang et al., 2019; Shi et al., 2020a). It is an effective way to promote the transportation of ions and electrons by fabricating low-tortuosity electrode.

The three-dimensional ordered porous carbon (3D-OPC) possesses low tortuosity and open pores (Qiao et al., 2019; Zhou et al., 2019). When used as an electrode material, it can not only provide fast electron transport path, but also significantly shorten ion diffusion length to 
achieve fast kinetic performance (Figure 1a). In addition, 3DOPC effectively suppresses the agglomeration of nanoparticles and maintains a large specific surface area (Zhao et al., 2015). However, the controllable preparation of 3D-OPC is still a challenge. In this review, we summarize the controllable preparation methods for 3D-OPC, and the latest research progress of $3 \mathrm{D}-\mathrm{OPC}$ in energy storage and conversion applications.

\section{PREPARATION METHODS}

The methods to prepare 3D-OPC can be divided into templateassisted methods and non-template approaches. Based on the different types of templates, the template-assisted methods include hard-templating method (Masuda and Fukuda, 1995; Li X. et al., 2018), ice-templating method (Roberts et al., 2014; Zhai et al., 2017), soft-templating method (Peng et al., 2019), and self-templating method (Feng and Yin, 2018). However, the soft-templating method normally lack of controllability, which is difficult to form a stable structure with vertical channels. Herein, we focus on the research progress of the other three template-assisted preparation methods for 3D-OPC.

\section{Ice-Templating Synthesis}

The ice-templating method is based on the solid phase produced in the process of phase separation, followed by removing solidified solvent with the solid phase frame retained (Shao et al., 2020). The structures produced by ice-templating method can be easily regulated. Moreover, this method is applicable to a wide range of materials, such as polymers, carbon aerogels and so on. According to different precursors, carbon aerogels can be divided into graphene aerogels, carbon nanotube-based aerogels, biomass-derived aerogels and so on (Shahbazi et al., 2020). Graphene oxide (GO) aerogels with good physical and chemistry properties is one of the most widely studied carbonbased aerogels. Wang et al. first proposed a freeze-drying method followed by a reduction process to prepare the reduced graphene oxide (rGO) aerogels (Wang and Ellsworth, 2009). Since then, the graphene aerogels prepared by the ice-templating method have been extensively studied. As a typical example, Zhang et al. added ethanol to the GO suspension, which was then transferred to a polytetrafluoroethylene (PTFE) mold for the subsequent rapid-freeze process in liquid nitrogen (Zhang P. et al., 2017). Finally, a long vertical graphene film was produced by freeze-drying and annealing. Ethanol is used as anti-freeze agent to reduce the freezing point, which has a significant influence on the crystallization behavior of ice. In addition, Wang et al. prepared a GO aerogel with a radially aligned structure by bidirectional freeze-casting method (Wang C. et al., 2018). During the freezing process, ordered porous GO aerogel was formed with a continuous $3 \mathrm{D}$ network. To study freezing/assembling mechanism, the authors added different additives, such as ethanol, chitosan or cellulose nanofibers to the aqueous solution of GO. It is revealed that different additives affected the interaction between ice crystals and GO nanosheets, resulting in the ice crystals with different shapes. Furthermore,
Mochizuki et al. reported vertical aligned rGO electrodes through electrodeposition of GO to form rGO and subsequent freezedrying process (Figure 1b; Mochizuki et al., 2019). This method can control the film thickness and pore size to achieve fast charge storage and low resistance. In addition, the pore size can be controlled in the range of $10-100 \mu \mathrm{m}$ by adjusting the freezing rate.

\section{Hard-Templating Synthesis}

The hard template not only has high stability, but also can control morphology and increase specific surface area of catalysts. There are numerous hard templates, such as salt (Zhang et al., 2020), metal (Cao et al., 2011), and silica (Byeon et al., 2020), available to prepare carbon materials. For example, Kim et al. used various-sized silica nanoparticles as template which was subsequently removed by $1 \mathrm{M} \mathrm{NaOH}$ to obtain mesoporous nitrogen (N)-doped carbon (Byeon et al., 2020). Kyotani et al. (1988) first proposed that carbon materials can be synthesized via hard-templating method in 1988. Further, the next breakthrough was achieved by Kyotani et al. (1995), who synthesized carbon nanotubes with a uniform length and diameter by using anodic aluminum oxide (AAO) as a hard template in 1995. The porous AAO template is a typical hard template with low cost and highly ordered nano-scale array channels. Since then, the preparation of various 3DOPCs through AAO template had become a research hotspot. Typically, Wang et al. used AAO as a hard template to prepare CNT-coated Sn nanoparticles with almost 100\% particle encapsulation and high filling uniformity by a chemical vapor deposition (CVD) process (Wang et al., 2009). Similarly, Zhou et al. prepared a sulfur-doped carbon nanotube cathode (SCNTs) by using sulfate-containing AAO templates (Figure 1c; Zhou et al., 2012). The specific surface area of S-CNTs with mesoporous structure reached $613 \mathrm{~m}^{2} \mathrm{~g}^{-1}$. The authors claimed that the mesoporous structures of S-CNTs can reduce cathode polarization, and promote ion transportation. Moreover, sulfur is confined in the walls of the nanotubes which can restrict the dissolution of formed polysulfides when used as cathode for Li-S battery.

\section{Self-Templated Synthesis}

Direct synthesis without additional template can significantly reduce cost in practical applications. Wood is a natural composite material composing vertical channels. After carbonization, its original features of low tortuous, open micro-channels and 3D layered porous structure can be maintained, which is suitable as building block for constructing ultra-thick electrodes. The wood-derived carbon material was first reported as electrode materials for supercapacitors in 2004 (Wu et al., 2004). Since then, the application of wood-derived materials have been further extended to batteries, electrocatalysis and so on. For example, $\mathrm{Li}$ et al. pre-carbonized wood slices at $260^{\circ} \mathrm{C}$ for $6 \mathrm{~h}$ in air atmosphere, then carbonized them at $1000^{\circ} \mathrm{C}$ for $6 \mathrm{~h}$ in $\mathrm{Ar}$ atmosphere, and finally activated the carbonized slices in $\mathrm{CO}_{2}$ atmosphere at $800^{\circ} \mathrm{C}$ ( $\mathrm{Li}$ et al., 2017). During the process, $\mathrm{CO}_{2}$ reacted with the amorphous carbon in carbonized wood to produce porous structure. The activated wood has an anisotropic 
a
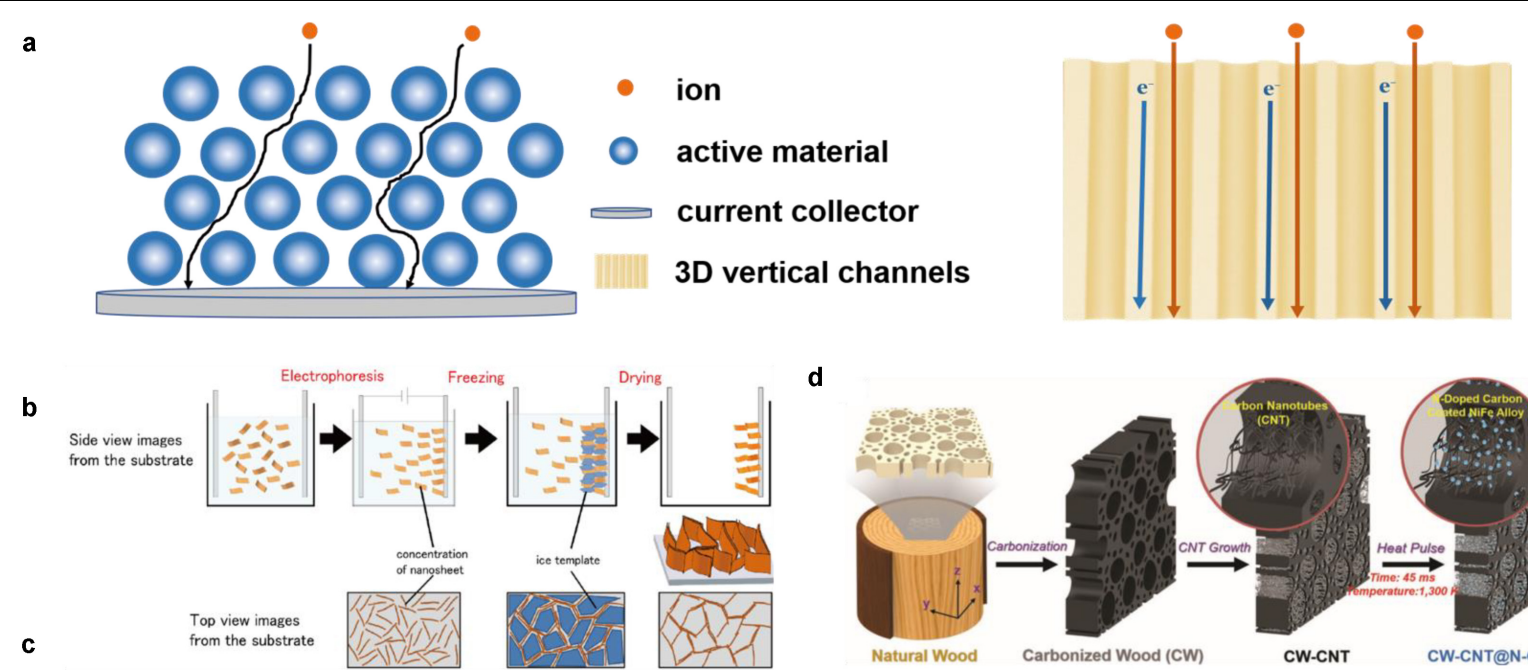

d
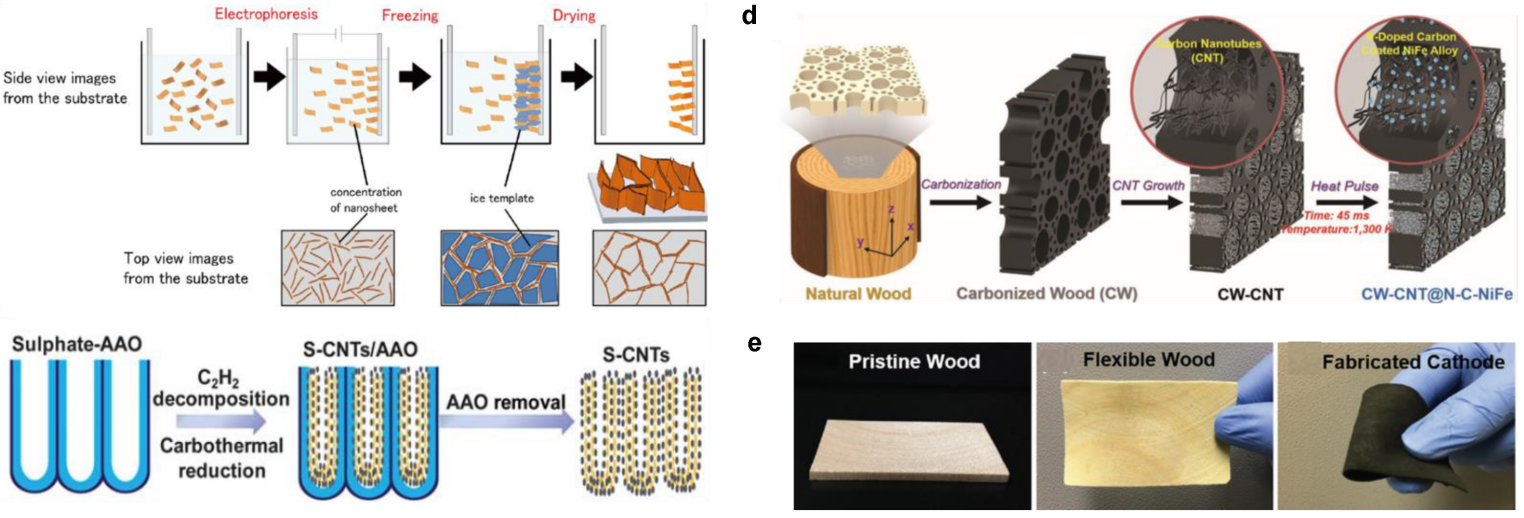

FIGURE 1 | (a) Schematic illustration of the different configurations of electrode materials: fabricate electrodes by slurry-casting process (left) and 3D-OPC electrodes (right). (b) Formation mechanism of the vertically aligned film by electrophoresis and freeze-drying (source: Mochizuki et al., 2019). Reproduced by permission of American Chemical Society https://doi.org/10.1021/acsaem.8b01478. (c) Thermal decomposition of $\mathrm{C}_{2} \mathrm{H}_{2}$ in a sulfate-containing $\mathrm{AAO}$ template and the formation of S-CNTs after AAO removal (source: Zhou et al., 2012). Reproduced by permission of Royal Society of Chemistry https://doi.org/

10.1039/C2EE22294A. (d) The fabrication process of the CW-CNT@N-C-NiFe electrode (source: Li Y. et al., 2018). Reproduced by permission of WILEY-VCH Verlag GmbH \& Co. KGaA, Weinheim https://doi.org/10.1002/aenm.201801289. (e) Photos of the pristine wood, the flexible wood, and the flexible cathode with the CNT coating and Ru (source: Xu et al., 2018). Reproduced by permission of Royal Society of Chemistry https://doi.org/10.1039/C8EE01468J.

structure and excellent deformation resistance. After that, rGO and $S$ were filled into the microchannels of the carbonized wood to achieve a high sulfur mass load. Furthermore, Wu et al. used $\mathrm{H}_{2}$ as the reducing gas, ethylene as the carbon source and Ar gas as the carrying gas to in situ grow CNTs in carbonized wood channels by CVD method (Figure 1d; Wu et al., 2019). The specific surface area of the electrode was increased to $537.9 \mathrm{~m}^{2} \mathrm{~g}^{-1}$ because of the introduction of CNTs. Then, the authors improved the hydrophilicity of the material after treating them using a mixed solution containing $\mathrm{HNO}_{3}$ and $\mathrm{H}_{2} \mathrm{SO}_{4}$, achieving the reduction of contact angle from 144 to $27.1^{\circ}$. Lignin, cellulose and hemicellulose are important components of wood. Xu et al. used sulfites to remove lignin which makes wood rigidity (Xu et al., 2019). Meanwhile, hemicellulose and cellulose could be dissolved by soaking in a basic solution containing sulfites, which made the treated wood more flexible (Figure 1e). Later, the surface of the flexible carbonized wood was coated with ruthenium-decorated CNT network $(\mathrm{CNT} / \mathrm{Ru})$ by vacuum-assisted method. Based on the Xu's research, Chen et al. reported a carbonized sponge with a high compressibility of $\sim 80 \%$ and high fatigue resistance (10,000 cycles at $50 \%$ strain), which was prepared by a wood template (Chen et al., 2018). In this work, more lignin and hemicellulose inside wood were removed by a treatment of $\mathrm{H}_{2} \mathrm{O}_{2}$. A subsequent carbonization leads to a sponge with unique lamellar structure.

\section{APPLICATIONS OF ENERGY STORAGE AND CONVERSION}

\section{Electrocatalysis}

Noble metal-based catalysts (e.g., Pt and Ir) are efficient electrocatalysts for oxygen evolution reaction (OER), oxygen reduction reaction (ORR) and hydrogen evolution reaction (HER). However, scarcity and valuableness greatly restrict their commercial applications (Deng et al., 2019; Liu et al., 2019, 2020). Therefore, it is desirable to develop inexpensive and efficient alternatives. Ordered porous carbon with vertical channels is a promising candidate, which has advantages of fast ion diffusion and electron transport.

Recently, 3D wood-based materials have aroused numerous researchers' interest. $\mathrm{Li}$ et al. demonstrated the core-shell nitrogen doped, few-graphene-layer-encapsulated nickel iron alloy nanoparticles (N-C-NiFe) in the carbonized wood with CNTs (N-C-NiFe@CW-CNT) by thermal-pulse method (Li Y. et al., 2018). The N-C-NiFe was anchored on CNTs uniformly (Figures 2a-d). Low-tortuosity microchannels were favor to hydrogen release and electrolyte penetration, therefore, N-CNiFe@CW-CNT showed good electrochemical performance of HER with a small Tafel slop $\left(52.8 \mathrm{mV} \mathrm{dec}^{-1}\right)$, low overpotential $\left(179 \mathrm{mV}\right.$, at $10 \mathrm{~mA} \mathrm{~cm}^{-2}$ ) and good cycling stability (Figure 2e). Inspired by this, Yang et al. designed a $\mathrm{CoFeP}_{\mathrm{x}}$ nano-catalyst with thin iron shells in wood wall $\left(\mathrm{CoFeP}_{\mathrm{x}} / \mathrm{c}\right.$-wood) by making 

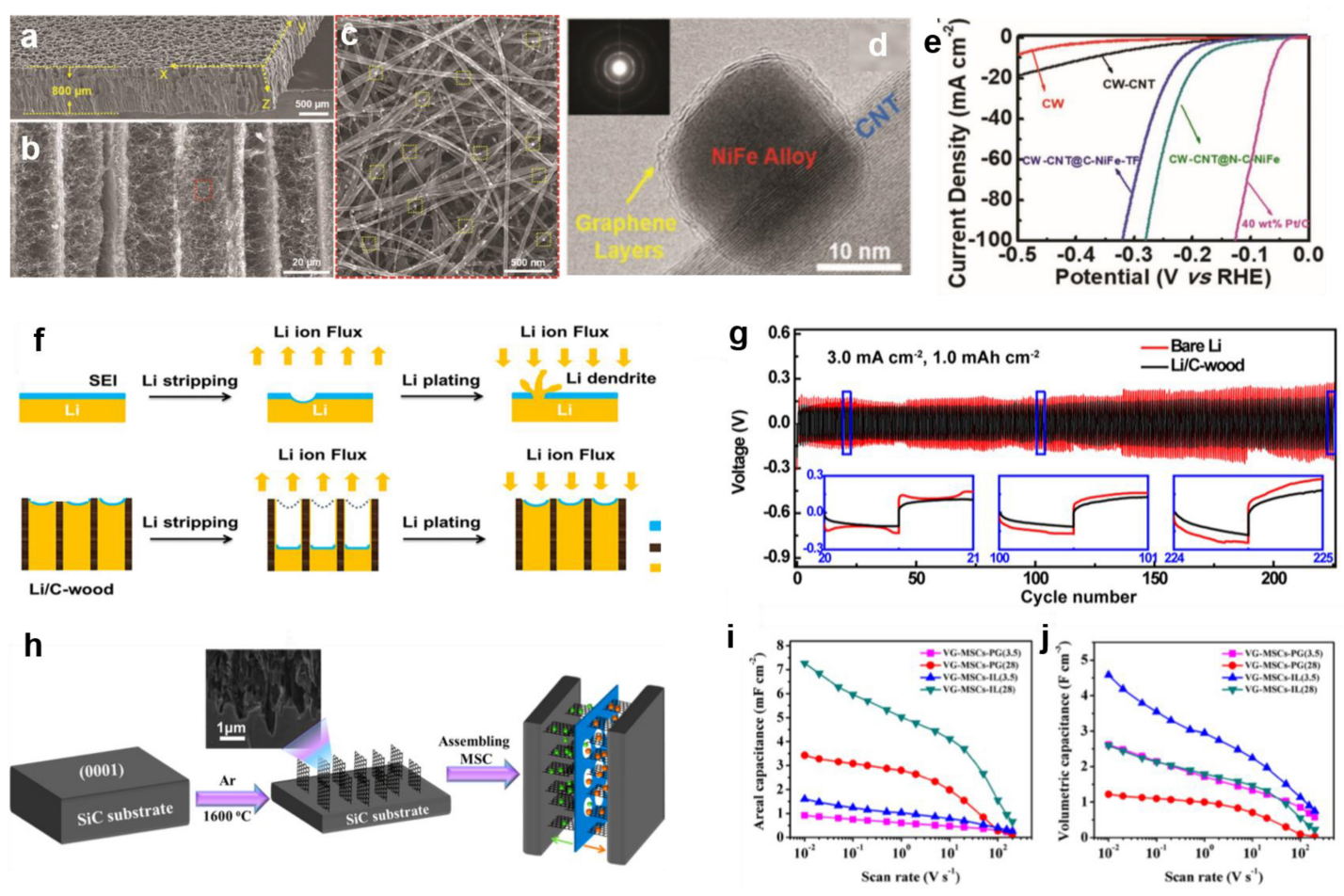

FIGURE 2 | (a-d) Morphology of the CW-CNT@N-C-NiFe. (a-c): SEM images of CW-CNTs@N-C-NiFe (d)TEM image of CW-CNTs@N-C-NiFe, it shows the NiFe alloy nanoparticle which is inset in CNT and encapsulated by the few graphene layers) (e) LSV curves of the CW, CW-CNTs, CW-CNTs@C-NiFe-NF, CW-CNTs@N-C-NiFe, and commercial 40 wt\% Pt/C electrodes (source: Li Y. et al., 2018). Reproduced by permission of WILEY-VCH Verlag GmbH \& Co. KGaA, Weinheim https://doi.org/10.1002/aenm.201801289. (f) Li stripping/plating of bare Li metal electrodes and Li/C-wood electrodes. (g) Voltage profiles of bare Li metal and Li/c-wood cell at $3 \mathrm{~mA} \cdot \mathrm{cm}^{-2}$, Li/c-wood cells exhibited smaller voltage and long-term cycling stability (source: Zhang Y. et al., 2017). Reproduced by permission of American Chemical Society https://doi.org/10.1073/pnas.1618871114. (h) VG films derived from the SiC substrates to form VG-MSCs. (i,j) Areal/volumetric capacitance of different VG-MSCs (PG means using $\mathrm{H}_{2} \mathrm{SO}_{4} / \mathrm{PVA}$ as gel electrolyte, IL means using EMIMBF 4 as liquid electrolyte) (source: Zheng et al., 2017). Reproduced by permission of American Chemical Society https://doi.org/10.1021/acsnano.7b00553.

use of the steam pressure difference between Fe and Co at high temperature (Yang et al., 2019). The thin iron shell $(\sim 2 \mathrm{~nm})$ as the protective layer of $\mathrm{CoFeP}_{\mathrm{x}}$ greatly improved the durability of catalyst without sacrificing the activity of the catalyst due to geometric effects of the thin coating layer. Compared to $\mathrm{CoP}_{\mathrm{x}}$ and $\mathrm{FeP}_{\mathrm{x}}, \mathrm{CoFeP}_{\mathrm{x}}$ showed a better OER electrochemical performance with lowest overpotential $(323 \mathrm{mV}$ at $10 \mathrm{~mA}$ $\mathrm{cm}^{-2}$ ) and Tafel slope $\left(58 \mathrm{mV} \mathrm{dec}{ }^{-1}\right)$. Besides, $\mathrm{CoFeP}_{\mathrm{x}} / \mathrm{c}$-wood possessed excellent cycling stability of $50 \mathrm{~h}$.

\section{Batteries}

Rechargeable batteries, such as lithium-ion batteries (Zhang et al., 2018; Deng et al., 2020a,b; Huang et al., 2020), sodium-ion batteries (Gao et al., 2020; Jin et al., 2020; Shi et al., 2020b,c; Wang et al., 2020) and metal-air batteries (Shi et al., 2019; Wu F. et al., 2020), have been intensively investigated recently. Due to the long cycling life, high voltage and energy density, lithium-ion batteries are widely used in electronic devices and other fields (Yan et al., 2019; Deng et al., 2020b).

Li metal anode undergoes large volume change during Li ion intercalation/deintercalation, which destroys the solid electrolyte interphase (SEI), making fresh Li metal exposed to electrolyte and leads to excessive growth of SEI. Zhang Y. et al. (2017) injected the molten lithium metal into the channels of carbonized wood to form a Li metal/carbonized wood (lithium/c-wood) electrode, in which the $\mathrm{ZnO}$ coating made the interface between c-wood and Li more wettable. Moreover, c-wood with 3D porous conductive frame structure is an ideal host for lithium metal, which can adapt to the volume change of lithium metal during the long cycle (Figure 2f). In addition, compared to bare $\mathrm{Li}$ metal, $\mathrm{Li} / \mathrm{c}$-wood electrodes in symmetrical batteries had a lower overpotential ( $90 \mathrm{mV}$ at $3 \mathrm{~mA} \mathrm{~cm}{ }^{-2}$ ), better cycling performance $\left(150 \mathrm{~h}\right.$ at $\left.3 \mathrm{~mA} \mathrm{~cm}{ }^{-2}\right)$ and more stable stripping/plating profiles. Even at a high current density of $3 \mathrm{~mA} \mathrm{~cm}{ }^{-2}$, the $\mathrm{Li} / \mathrm{c}$-wood electrodes in the commercial electrolyte achieved long-term cyclic stability (Figure 2g). Similarly, Xue et al. (2018) assembled continuous silver nanowires onto an interconnected 3D graphene network (3D Ag-GN) by ice-templating method, and designed a layered 3D porous structure as a host for the lithium metal composite anode. The hierarchical networks not only provided ultrafast electron transport channels and non-nucleation barrier sites to limit lithium deposition, but also had excellent mechanical strength to support lithium deposition. Moreover, 3D Ag-GN could work over 1000 plating/stripping cycles with low overpotential (at $40 \mathrm{~mA} \mathrm{~cm}{ }^{-2}$ ) in a symmetric cell. As the thickness of the electrode increased, the dynamics of ion 
transport were hindered, and the rate performance of the lithiumion batteries decreased. Zhang et al. (2019) used magnetite $\left(\mathrm{Fe}_{3} \mathrm{O}_{4}\right)$ with high theoretical capacity to produce aligned $\mathrm{Fe}_{3} \mathrm{O}_{4} / \mathrm{GO}$ electrodes with fast ion transmission channels by icetemplating method. Besides, they proved that $\mathrm{Fe}_{3} \mathrm{O}_{4} / \mathrm{GO}$ had a fast ion transfer kinetics by galvanostatic intermittent titration technique (GITT), electrochemical impedance spectroscopy (EIS), and cyclic voltammetry (CV).

\section{Supercapacitors}

Supercapacitors is one of the most promising energy storage devices due to long lifetime, high power density, and fast charge/discharge rates (Xu et al., 2017, 2018). However, traditional supercapacitor electrode materials (e.g., activated carbon, carbonized derived carbon, and carbon nanotubes) normally have a long ion-diffusion path. Moreover, the presence of binder in powder-based electrode materials covers a part of the active sites, which reduces energy density. Electrode with vertical channels can provide fast mass transportation. Zheng et al. (2017) designed vertically aligned graphene (VG) nanocrystals by decomposing $\mathrm{SiC}$ matrix at high temperature, which used as electrode materials for micro-supercapacitors (MSCs) (Figure 2h). The VG nanocrystals micro-supercapacitors (VG-MSCs) had high areal and volumetric capacitance, and fast frequency response. More importantly, it showed high power density in gel electrolyte $\left(\sim 15 \mathrm{~W} \mathrm{~cm}^{-3}\right)$ and ionic liquid $(\sim 61 \mathrm{~W}$ $\mathrm{cm}^{-3}$ ) (Figures 2i,j). In addition, Chen et al. designed an all-wood-structured asymmetric supercapacitor (ASC) using a $\mathrm{MnO}_{2}$ /wood carbon as cathode, an activated wood carbon as anode, and a wood membrane as separator (Chen et al., 2017). Remarkably, a high mass load of active material can be achieved by increasing electrode thickness or extending electrodeposition time of $\mathrm{MnO}_{2}$. The prepared electrode materials maintained the unique anisotropy structure of the wood and highly exposure active sites, allowing the electrolyte ions to pass directly through the channels, showing high ionic conductivity. The ASC retained $\sim 0.6 \mathrm{~mW} \mathrm{~h} \mathrm{~cm}{ }^{-2}\left(5.7 \mathrm{~W} \mathrm{~h} \mathrm{~kg}{ }^{-1}\right)$ at a power density of $24440 \mathrm{~mW} \mathrm{~cm}^{-2}\left(107.7 \mathrm{~W} \mathrm{~kg}^{-1}\right)$, which can light up a lightemitting diode (LED) after pre-charging to $1.8 \mathrm{~V}$. Furthermore, Wang Y. et al. (2018) applied $\mathrm{Co}(\mathrm{OH})_{2} @ \mathrm{CW}$ as cathode of an all-solid-state asymmetric supercapacitors, which possessed high energy/power density, and maintained $85 \%$ capacitance retention after 10000 charge/discharge cycles.

\section{CONCLUSION}

The versatility and availability of the 3D-OPC with high specific surface area, highly ordered channels, low tortuosity and open pores have been demonstrated in various applications in energy storage and conversion. In this review, we outlined the latest development on the rational design and controllable preparation of 3D-OPC and their composites for applications of electrocatalysis, batteries and supercapacitors. The strategies to achieve controllable synthesis of 3D-OPC can be classified as follows. Firstly, a hard template with appropriate size and morphology can be selected to synthesize electrode materials. Secondly, in ice-templated approach, the electrode thickness and pore size can be controlled through modulating the crystallization behavior of ice by adding various additives (e.g., ethanol, chitosan, or cellulose nanofibers), and adjusting the freezing rate, which achieve high conductivity, shorten ion diffusion length and large specific surface area. Thirdly, carbonization temperature and calcination atmosphere control the shrinkage rate as well as the consequent aperture changes of carbonized wood, which play an essential role in the formation of internal pores and surface morphology of samples.

Although the 3D-OPC or their composites with ultrafast electron/ion transport path, abundant active sites, and fast reaction kinetics showed superior electrochemical performance when applied in energy storage devices, it still has several challenges. First, the methods based on hard templates inevitably involve the corrosive solution in post-processing step, which largely increases the cost and is not suitable to industrialization. Second, the controllable growth of active materials on the 3DOPC is still a challenge, since uneven distribution of reactants in channels normally results in asymmetrical nucleation and growth of active materials. Third, the relation between morphology, pore size, structure of the $3 \mathrm{D}-\mathrm{OPC}$ and electrochemical performance is vague and imprecise.

In spite of the vast research and progress that were discussed, there are still many opportunities for further advancement of 3D-OPC. First, the influence of the penetrability of vertical channels (the diffusion of adjacent channels) on electrochemical performance could be further studied. Second, the other active materials, such as perovskite, single atom, can be loaded on the $3 \mathrm{D}-\mathrm{OPC}$ to improve the performance for photocatalysis, electrocatalysis, and so on. Third, it is significant to proceed the computational simulation about the relation between pore size of vertical channels and electrochemical performance. Moreover, there are still some other methods may be explored for preparing 3D-OPC, such as template-free method, biomassderived method, the assembly of $\mathrm{sp}^{2}$-hybridized carbon. It is also promising to explore the applications of 3D-OPC in gas separation, dye degradation, water purification, and capacitive deionization (CDI). Therefore, tremendous explorations and optimizations of the 3D-OPC and their composites are indispensable in the future work.

\section{AUTHOR CONTRIBUTIONS}

JF, DZ, XG, WQ, WS, WL, FW, and XC contributed to the discussion and writing in this work. All authors listed have made a substantial, direct and intellectual contribution to the work, and approved it for publication.

\section{FUNDING}

This work was supported by National Natural Science Foundation of China (Grant Nos. 51702286, 51972286, and 21905246) and Zhejiang Provincial Natural Science Foundation of China (LR19E020003 and LQ20B010011), and the China Postdoctoral Science Foundation Funded Project (2019M660144 and 2020T130596). 


\section{REFERENCES}

Byeon, A., Cho, J., Kim, J., Chae, K., Park, H., Hong, S., et al. (2020). Highyield electrochemical hydrogen peroxide production from an enhanced twoelectron oxygen reduction pathway by mesoporous nitrogen-doped carbon and manganese hybrid electrocatalysts. Nanoscale Horiz. 5, 832-838. doi: 10.1039/ c9nh00783k

Cao, X., Shi, Y., Shi, W., Lu, G., Huang, X., Yan, Q., et al. (2011). Preparation of novel 3D graphene networks for supercapacitor applications. Small 7, 31633168. doi: 10.1002/smll.201100990

Cao, X., Tan, C., Sindoro, M., and Zhang, H. (2017). Hybrid micro/nano-structures derived from metal-organic frameworks: preparation and applications in energy storage and conversion. Chem. Soc. Rev. 46, 2660-2677. doi: $10.1039 / \mathrm{c} 6 \mathrm{cs} 00426 \mathrm{a}$

Cao, X., Yin, Z., and Zhang, H. (2014). Three-dimensional graphene materials: preparation, structures and application in supercapacitors. Energy Environ. Sci. 7, 1850-1865. doi: 10.1039/c4ee00050a

Chen, C., Song, J., Zhu, S., Li, Y., Kuang, Y., Wan, J., et al. (2018). Scalable and sustainable approach toward highly compressible, anisotropic, lamellar carbon sponge. Chem 4, 544-554. doi: 10.1016/j.chempr.2017.12.028

Chen, C., Zhang, Y., Li, Y., Dai, J., Song, J., Yao, Y., et al. (2017). All-wood, low tortuosity, aqueous, biodegradable supercapacitors with ultra-high capacitance. Energy Environ. Sci. 10, 538-545. doi: 10.1039/c6ee03716j

Deng, S., He, Z., Liu, B., Yang, L., Wang, X., Shen, S., et al. (2020a). Synergy of ion doping and spiral array architecture on Ti2Nb10O29: a new way to achieve high-power electrodes. Adv. Funct. Mater. 30:2002665. doi: 10.1002/ adfm. 202002665

Deng, S., Zhu, H., Wang, G., Luo, M., Shen, S., Ai, C., et al. (2020b). Boosting fast energy storage by synergistic engineering of carbon and deficiency. Nat. Commun. 11:132. doi: 10.1038/s41467-019-13945-1

Deng, S., Luo, M., Ai, C., Zhang, Y., Liu, B., Huang, L., et al. (2019). Synergistic doping and intercalation: realizing deep phase modulation on MoS2 arrays for high-efficiency hydrogen evolution reaction. Angew. Chem. Int. Ed. 58, 16289-16296. doi: 10.1002/anie.201909698

Feng, J., and Yin, Y. (2018). Self-templating approaches to hollow nanostructures. Adv. Mater. 31:1802349. doi: 10.1002/adma.201802349

Gao, X., Shi, W., Ruan, P., Feng, J., Zheng, D., Yu, L., et al. (2020). Ultrathin carbon boosted sodium storage performance in aqueous electrolyte. Funct. Mater. Lett. 13, 2030002-2030010. doi: 10.1142/s1793604720300029

Huang, L., Li, J., Liu, B., Li, Y., Shen, S., Deng, S., et al. (2020). Electrode design for lithium-sulfur batteries: problems and solutions. Adv. Funct. Mater. 30:1910375. doi: 10.1002/adfm.201910375

Jin, T., Han, Q., and Jiao, L. (2020). Binder-free electrodes for advanced sodium-ion batteries. Adv. Mater. 32:e1806304. doi: 10.1002/adma.201806304

Kyotani, T., Sonobe, N., and Tomita, A. (1988). Formation of highly orientated graphite from polyacrylonitrile by using a two-dimensional space between montmorillonite lamellae. Nature 331, 331-333. doi: 10.1038/33 $1331 \mathrm{a} 0$

Kyotani, T., Tsai, L.-F., and Tomita, A. (1995). Formation of ultrafine carbon tubes by using an anodic aluminum oxide film as a template. Chem. Mater. 7, 1427-1428. doi: 10.1021/cm00056a001

Li, C., Li, Q., Kaneti, Y., Hou, D., Yamauchi, Y., and Mai, Y. (2020). Self-assembly of block copolymers towards mesoporous materials for energy storage and conversion systems. Chem. Soc. Rev. 49, 4681-4736. doi: 10.1039/d0cs00021c

Li, W., Fang, R., Xia, Y., Zhang, W., Wang, X., Xia, X., et al. (2019). Multiscale porous carbon nanomaterials for applications in advanced rechargeable batteries. Batter. Supercaps 2, 9-36. doi: 10.1002/batt.201800067

Li, X., Guan, B., Gao, S., and Lou, X. (2018). A general dual-templating approach to biomass-derived hierarchically porous heteroatom-doped carbon materials for enhanced electrocatalytic oxygen reduction. Environ. Sci. Technol. 12:648. doi: 10.1039/C8EE02779J

Li, Y., Gao, T., Yao, Y., Liu, Z., Kuang, Y., Chen, C., et al. (2018). In situ "chainmail catalyst" assembly in low-tortuosity, hierarchical carbon frameworks for efficient and stable hydrogen generation. Adv. Energy Mater. 8:1801289. doi: 10.1002/aenm.201801289

Li, Y., Fu, K., Chen, C., Luo, W., Gao, T., Xu, S., et al. (2017). Enabling highareal-capacity lithium-sulfur batteries: designing anisotropic and low-tortuosity porous architectures. ACS Nano 11, 4801-4807. doi: 10.1021/acsnano.7b01172
Liu, W., Yin, R., Xu, X., Zhang, L., Shi, W., and Cao, X. (2019). Structural engineering of low-dimensional metal-organic frameworks: synthesis, properties, and applications. Adv. Sci. 6:1802373. doi: 10.1002/advs.201802373

Liu, W., Yu, L., Yin, R., Xu, X., Feng, J., Jiang, X., et al. (2020). Non-3d metal modulation of a $2 \mathrm{D} \mathrm{Ni-Co}$ heterostructure array as multifunctional electrocatalyst for portable overall water splitting. Small 16:1906775. doi: 10. 1002/smll.201906775

Masuda, H., and Fukuda, K. (1995). Ordered metal nanohole arrays made by a two-step replication of honeycomb structures of anodic alumina. Science 268, 1466-1468. doi: 10.1126/science.268.5216.1466

Mochizuki, D., Tanaka, R., Makino, S., Ayato, Y., and Sugimoto, W. (2019). Vertically aligned reduced graphite oxide nanosheet film and its application in a high-speed charge/discharge electrochemical capacitor. ACS Appl. Energy Mater. 2, 1033-1039. doi: 10.1021/acsaem.8b01478

$\mathrm{Ni}$, J., and Li, L. (2020). Cathode architectures for rechargeable ion batteries: progress and perspectives. Adv. Mater. 32:2000288. doi: 10.1002/adma. 202000288

Peng, H., Yao, B., Wei, X., Liu, T., Kou, T., Xiao, P., et al. (2019). Pore and heteroatom engineered carbon foams for supercapacitors. Adv. Energy Mater. 9:1803665. doi: 10.1002/aenm.201803665

Qiao, Y., Han, R., Pang, Y., Lu, Z., Zhao, J., Cheng, X., et al. (2019). 3D wellordered porous phosphorus doped carbon as an anode for sodium storage: structure design, experimental and computational insights. J. Mater. Chem. A 7, 11400-11407. doi: 10.1039/c9ta02268f

Roberts, A., Li, X., and Zhang, H. (2014). Porous carbon spheres and monoliths: morphology control, pore size tuning and their applications as Li-ion battery anode materials. Chem. Soc. Rev. 43, 4341-4356. doi: 10.1039/c4cs00071d

Shahbazi, M.-A., Ghalkhani, M., and Maleki, H. (2020). Directional freeze-casting: a bioinspired method to assemble multifunctional aligned porous structures for advanced applications. Adv. Eng. Mater. 22:2000033. doi: 10.1002/adem. 202000033

Shao, G., Hanaor, D., Shen, X., and Gurlo, A. (2020). Freeze casting: from lowdimensional building blocks to aligned porous structures-A review of novel materials, methods, and applications. Adv. Mater. 32:1907176. doi: 10.1002/ adma.201907176

Shi, B., Shang, Y., Pei, Y., Pei, S., Wang, L., Heider, D., et al. (2020a). Low tortuous, highly conductive, and high-areal-capacity battery electrodes enabled by through-thickness aligned carbon fiber framework. Nano Lett. 20, 55045512. doi: 10.1021/acs.nanolett.0c02053

Shi, W., Gao, X., Mao, J., Qian, X., Liu, W., Wu, F., et al. (2020b). Exploration of energy storage materials for water desalination via next-generation capacitive deionization. Front. Chem. 8:415. doi: 10.3389/fchem.2020.00415

Shi, W., Liu, X., Deng, T., Huang, S., Ding, M., Miao, X., et al. (2020c). Enabling superior sodium capture for efficient water desalination by a tubular polyaniline decorated with prussian blue nanocrystals. Adv. Mater. 2020:1907404. doi: 10. 1002/adma.201907404

Shi, W., Mao, J., Xu, X., Liu, W., Zhang, L., Cao, X., et al. (2019). An ultra-dense NiS2/reduced graphene oxide composite cathode for highvolumetric/gravimetric energy density nickel-zinc batteries. J. Mater. Chem. A 7, 15654-15661. doi: 10.1039/c9ta04900b

Ullah, S., Hasan, M., Ta, H., Zhao, L., Shi, Q., Fu, L., et al. (2019). Synthesis of doped porous 3D graphene structures by chemical vapor deposition and its applications. Adv. Funct. Mater. 29:1904457. doi: 10.1002/adfm.201904457

Wang, B., Cheng, Y., Su, H., Cheng, M., Li, Y., Geng, H., et al. (2020). Boosting transport kinetics of cobalt sulfides yolk-shell spheres by anion doping for advanced lithium and sodium storage. ChemSusChem 13, 1-9. doi: 10.1002/ cssc. 202001261

Wang, C., Chen, X., Wang, B., Huang, M., Wang, B., Jiang, Y., et al. (2018). Freezecasting produces a graphene oxide aerogel with a radial and centrosymmetric structure. ACS Nano 12, 5816-5825. doi: 10.1021/acsnano.8b01747

Wang, Y., Lin, X., Liu, T., Chen, H., Chen, S., Jiang, Z., et al. (2018). Woodderived hierarchically porous electrodes for high-performance all-solid-state supercapacitors. Adv. Funct. Mater. 28:1806207. doi: 10.1002/adfm.201806207

Wang, J., and Ellsworth, M. (2009). Graphene aerogels. ECS Trans. 19, 241-247. doi: $10.1149 / 1.3119548$

Wang, X., Wang, T., Borovilas, J., He, X., Du, S., and Yang, Y. (2019). Verticallyaligned nanostructures for electrochemical energy storage. Nano Res. 12, 20022017. doi: $10.1007 / \mathrm{s} 12274-019-2392-\mathrm{x}$ 
Wang, Y., Wu, M., Jiao, Z., and Lee, J. (2009). Sn@CNT and Sn@C@CNT nanostructures for superior reversible lithium ion storage. Chem. Mater. 21, 3210-3215. doi: 10.1021/cm900702d

Wu, C., Zhang, S., Wu, W., Xi, Z., Zhou, C., Wang, X., et al. (2019). Carbon nanotubes grown on the inner wall of carbonized wood tracheids for highperformance supercapacitors. Carbon 150, 311-318. doi: 10.1016/j.carbon.2019. 05.032

Wu, F.-C., Tseng, R.-L., Hu, C.-C., and Wang, C.-C. (2004). Physical and electrochemical characterization of activated carbons prepared from firwoods for supercapacitors. J. Power Sources 138, 351-359. doi: 10.1016/j.jpowsour. 2004.06.023

Wu, F., Gao, X., Xu, X., Jiang, Y., Gao, X., Yin, R., et al. (2020). MnO2 nanosheetassembled hollow polyhedron grown on carbon cloth for flexible aqueous zinc-ion batteries. ChemSusChem 13, 1537-1545. doi: 10.1002/cssc.201903006

Wu, M., Liao, J., Yu, L., Lv, R., Li, P., Sun, W., et al. (2020). 2020 roadmap on carbon materials for energy storage and conversion. Chem. Asian J. 15, 995-1013. doi: 10.1002/asia.201901802

Wu, Q., Yang, L., Wang, X., and Hu, Z. (2020). Mesostructured carbon-based nanocages: an advanced platform for energy chemistry. Sci. China Chem. 63, 665-681. doi: 10.1007/s11426-020-9748-0

Xu, S., Chen, C., Kuang, Y., Song, J., Gan, W., Liu, B., et al. (2019). Flexible lithium$\mathrm{CO} 2$ battery with ultrahigh capacity and stable cycling. Environ. Sci. Technol. 11, 3231-3237. doi: 10.1039/C8EE01468J

Xu, X., Shi, W., Li, P., Ye, S., Ye, C., Ye, H., et al. (2017). Facile fabrication of three-dimensional graphene and metal-organic framework composites and their derivatives for flexible all-solid-state supercapacitors. Chem. Mater. 29, 6058-6065. doi: 10.1021/acs.chemmater.7b01947

Xu, X., Shi, W., Liu, W., Ye, S., Yin, R., Zhang, L., et al. (2018). Preparation of twodimensional assembled Ni-Mn-C ternary composites for high-performance all-solid-state flexible supercapacitors. J. Mater. Chem. A 6, 24086-24091. doi: $10.1039 / \mathrm{c} 8 \mathrm{ta} 06412 \mathrm{a}$

Xue, P., Liu, S., Shi, X., Sun, C., Lai, C., Zhou, Y., et al. (2018). A hierarchical silver-nanowire-graphene host enabling ultrahigh rates and superior long-term cycling of lithium-metal composite anodes. Adv. Mater. 30:1804165. doi: 10 . 1002/adma.201804165

Yan, Z., Jin, H., and Guo, J. (2019). Low-temperature synthesis of graphitic carbon-coated silicon anode materials. Carbon Energy 1, 246-252. doi: 10.1002/ cey 2.8

Yang, C., Cui, M., Li, N., Liu, Z., Hwang, S., Xie, H., et al. (2019). In situ iron coating on nanocatalysts for efficient and durable oxygen evolution reaction. Nano Energy 63:103855. doi: 10.1016/j.nanoen.2019.103855

Zhai, H., Xu, P., Ning, M., Cheng, Q., Mandal, J., and Yang, Y. (2017). A flexible solid composite electrolyte with vertically aligned and connected ionconducting nanoparticles for lithium batteries. Nano Lett. 17, 3182-3187. doi: 10.1021/acs.nanolett.7b00715

Zhang, F., Wei, M., Viswanathan, V., Swart, B., Shao, Y., Wu, G., et al. (2017). 3D printing technologies for electrochemical energy storage. Nano Energy 40, 418-431. doi: 10.1016/j.nanoen.2017. 08.037

Zhang, P., Li, J., Lv, L., Zhao, Y., and Qu, L. (2017). Vertically aligned graphene sheets membrane for highly efficient solar thermal generation of clean water. ACS Nano 11, 5087-5093. doi: 10.1021/acsnano.7b01965

Zhang, Y., Luo, W., Wang, C., Li, Y., Chen, C., Song, J., et al. (2017). High-capacity, low-tortuosity, and channel-guided lithium metal anode. Proc. Natl. Acad. Sci. U.S.A. 114, 3584-3589. doi: 10.1073/pnas.1618871114

Zhang, L., Liu, W., Shi, W., Xu, X., Mao, J., Li, P., et al. (2018). Boosting lithium storage properties of MOF derivatives through a wet-spinning assembled fiber strategy. Chemistry 24, 13792-13799. doi: 10.1002/chem.201802826

Zhang, X., Ju, Z., Housel, L., Wang, L., Zhu, Y., Singh, G., et al. (2019). Promoting transport kinetics in Li-ion battery with aligned porous electrode architectures. Nano Lett. 19, 8255-8261. doi: 10.1021/acs.nanolett.9b03824

Zhang, X., Zhang, S., Yang, Y., Wang, L., Mu, Z., Zhu, H., et al. (2020). A general method for transition metal single atoms anchored on honeycomblike nitrogen-doped carbon nanosheets. Adv. Mater. 32:1906905. doi: 10.1002/ adma.201906905

Zhao, Q., Wang, X., Liu, J., Wang, H., Zhang, Y., Gao, J., et al. (2015). Design and synthesis of three-dimensional hierarchical ordered porous carbons for supercapacitors. Electrochim. Acta 154, 110-118. doi: 10.1016/j.electacta.2014. 12.052

Zheng, S., Li, Z., Wu, Z., Dong, Y., Zhou, F., Wang, S., et al. (2017). High packing density unidirectional arrays of vertically aligned graphene with enhanced areal capacitance for high-power micro-supercapacitors. ACS Nano 11, 4009-4016. doi: 10.1021/acsnano.7b00553

Zhou, G., Wang, D.-W., Li, F., Hou, P.-X., Yin, L., Liu, C., et al. (2012). A flexible nanostructured sulphur-carbon nanotube cathode with high rate performance for Li-S batteries. Energy Environ. Sci. 5, 8901-8906. doi: 10.1039/c2ee $22294 \mathrm{a}$

Zhou, X., Chen, L., Zhang, W., Wang, J., Liu, Z., Zeng, S., et al. (2019). Threedimensional ordered macroporous metal-organic framework single crystalderived nitrogen-doped hierarchical porous carbon for high-performance potassium-ion Batteries. Nano Lett. 19, 4965-4973. doi: 10.1021/acs.nanolett. $9 \mathrm{~b} 01127$

Conflict of Interest: The authors declare that the research was conducted in the absence of any commercial or financial relationships that could be construed as a potential conflict of interest.

Copyright (C) 2020 Feng, Zheng, Gao, Que, Shi, Liu, Wu and Cao. This is an openaccess article distributed under the terms of the Creative Commons Attribution License (CC BY). The use, distribution or reproduction in other forums is permitted, provided the original author(s) and the copyright owner(s) are credited and that the original publication in this journal is cited, in accordance with accepted academic practice. No use, distribution or reproduction is permitted which does not comply with these terms. 